SciBX

\section{Going to bis-school}

\section{By Joanne Kotz, Senior Editor}

A team at Novartis AG has identified the first non-bisphosphonate inhibitors of farnesyl diphosphate synthase, ${ }^{1}$ an enzyme targeted by bonespecific bisphosphonates that are approved to treat benign and malignant bone diseases. The researchers believe the lower bone affinities of the new class of inhibitors could make the molecules better suited than bisphosphonates for cancer indications including soft tissue tumors.

However, safety could be a concern with these new compounds, as the target is part of the central mevalonate metabolic pathway that affects both cholesterol synthesis and protein prenylation ${ }^{2}$ (see Figure 1, "Targeting the mevalonate pathway").

In bone diseases, bisphosphonates decrease prenylation of critical signaling proteins in osteoclasts, leading to inhibition of osteoclast function. This is clearly useful in a disease like osteoporosis and also has utility in treating skeletal events related to bone metastases in cancer patients.

A case in point is Novartis' Zometa/Aclasta zoledronic acid, which is marketed for bone diseases and bone-related events in metastatic cancers. Additionally, the company is testing Zometa to treat soft tissue tumors, for which there is mounting evidence that bisphosphonates may have direct antitumor properties.

The drug is under FDA and EMA review as a breast cancer adjuvant to treat estrogen receptor-positive breast cancer in premenopausal women. In a Phase III trial in premenopausal women with early breast cancer, Zometa plus anastrozole or tamoxifen increased disease-free survival compared with either endocrine therapy alone. ${ }^{3}$

Figure 1. Targeting the mevalonate pathway. Jahnke et al. have identified allosteric inhibitors of farnesyl diphosphate synthase (FDPS; FPPS), an enzyme in the mevalonate pathway that is targeted by bisphosphonates to treat bone diseases. The new inhibitors, which lack the bone affinity of bisphosphonates, may enable better targeting of the enzyme in soft tissue tumors.

Three classes of drugs target the mevalonate pathway, which creates the metabolic building blocks for cholesterol synthesis and protein prenylation. Statins, which inhibit HMG-CoA reductase, are marketed by at least seven companies to treat metabolic, cardiovascular and neurological diseases. At least five companies market bisphosphonates to treat bone disease and bone-related events associated with metastatic cancer. Zarnestra tipifarnib, an inhibitor of one type of protein prenylation, is in clinical trials for cancer.
But the high bone affinity of bisphosphonates may limit their efficacy in soft tissue tumors.

Previous efforts to identify non-bisphosphonate inhibitors of farnesyl diphosphate synthase (FDPS; FPPS), also known as farnesyl pyrophosphate synthase, have been unsuccessful, primarily because the presence of a bisphosphonate group is essential to inhibit the target at the active site.

Now, the Novartis group led by Wolfgang Jahnke thinks it has found a work around-an allosteric binding pocket that does not require bisphosphonate groups. The researchers screened a 400-fragment library for in vitro binding to FPPS and identified several compounds that bound to the previously unknown pocket.

Jahnke is a senior scientific investigator and leading scientist at the Center for Proteomic Chemistry and Global Discovery Chemistry at the Novartis Institutes for Biomedical Research.

An in vitro NMR assay showed that none of the newly identified compounds had measurable binding to bone. Optimization of the fragments resulted in lead compounds that inhibited FPPS in vitro at nanomolar concentrations and inhibited protein prenylation in cells.

The study was published in Nature Chemical Biology.

The exciting part of the findings, according to Michael Rogers, is the potential for the new class of FPPS inhibitors to "open up all sorts of clinical possibilities, including treatment of soft tissue tumors."

Rogers is a professor of musculoskeletal pharmacology at The University of Aberdeen.

\section{Going soft}

Indeed, the next question is whether the FPPS inhibitors are now targeted too broadly.

Graham Russell, professor of musculoskeletal pharmacology at the University of Oxford, noted that two major classes of blockbuster drugs act in the mevalonate pathway: statins and bisphosphonates. Both classes
Marketed bisphosphonates: Zometa/Aclasta zoledronic acid from Novartis AG (NYSE:NVS; SIX:NOVN); Aredia pamidronate from Novartis; Fosamax alendronate from Merck \& Co. Inc. (NYSE:MRK); Nerixia nerodronate from Abiogen Pharma S.p.A.; Boniva ibandronate from Roche (SIX:ROG; OTCQX:RHHBY); and Actonel risedronate from

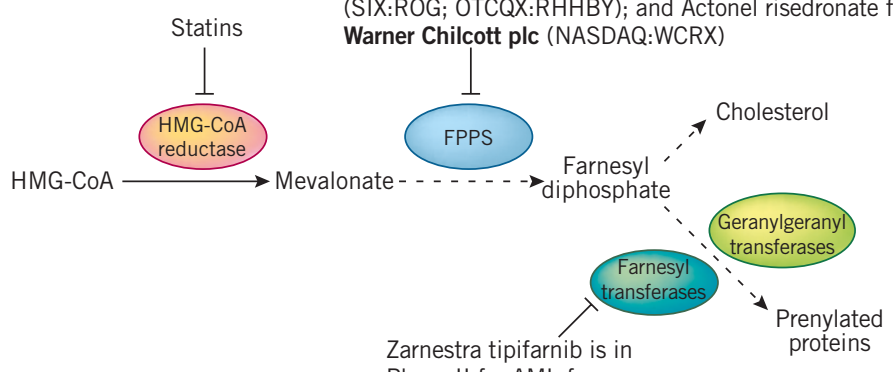

Johnson \& Johnson (NYSE:JNJ) 


\section{ANALYSIS}

\section{TARGETS \& MECHANISMS}

of drugs have high tissue selectivity-statins lower cholesterol via selective liver uptake, whereas bisphosphonates target bone- "which helps to ensure that these drugs don't have adverse effects on other organs," he said.

Farnesyl transferase inhibitors, which act downstream of FPPS and have a more limited effect on protein prenylation, have been generally well-tolerated by patients and have progressed to Phase II and Phase III testing in cancer.

Meanwhile, FPPS acts at a central node of the mevalonate pathway. "An FPPS inhibitor without bone affinity might be more toxic than inhibitors of farnesyl transferases," noted Said Sebti, director of the Drug Discovery Comprehensive Research Center and chair of the Department of Drug Discovery at the H. Lee Moffitt Cancer Center \& Research Institute.

Sebti, also a professor of oncological sciences at the University of South Florida, was a co-investigator in clinical trials of Zarnestra tipifarnib, a farnesyl transferase inhibitor from Johnson \& Johnson that is in Phase II trials for acute myeloid leukemia (AML).

For Novartis' new crop of FPPS inhibitors, Sebti suggested monitoring potential toxicity-related pathways downstream of the target, such as cholesterol synthesis, during preclinical testing.

After the inhibitors are optimized, both Russell and Rogers think in vivo testing is the next step, including breast cancer growth and metastasis models.

Jahnke told SciBX the compounds described in the paper are in early exploratory research for potential use in cancer.

Novartis declined to disclose the patent and licensing status of the work.

Kotz, J. SciBX 3(34); doi:10.1038/scibx.2010.1030

Published online Sept. 2, 2010

\section{REFERENCES}

1. Jahnke, W. et al. Nat. Chem. Biol.; published online Aug. 15, 2010; doi:10.1038/nchembio.421

Contact: Wolfgang Jahnke, Novartis Institutes for Biomedical Research, Basel, Switzerland

e-mail: wolfgang.jahnke@novartis.com

2. Russell, R.G.G. et al. Osteoporos. Int. 19, 733-759 (2008)

3. Gnant, M. et al. N. Engl. J. Med. 360, 679-691 (2009)

COMPANIES AND INSTITUTIONS MENTIONED

H. Lee Moffitt Cancer Center \& Research Institute, Tampa, Fla. Johnson \& Johnson (NYSE:JNJ), New Brunswick, N.J. Novartis AG (NYSE:NVS; SIX:NOVN), Basel, Switzerland The University of Aberdeen, Aberdeen, U.K.

University of Oxford, Oxford, U.K.

University of South Florida, Tampa, Fla. 